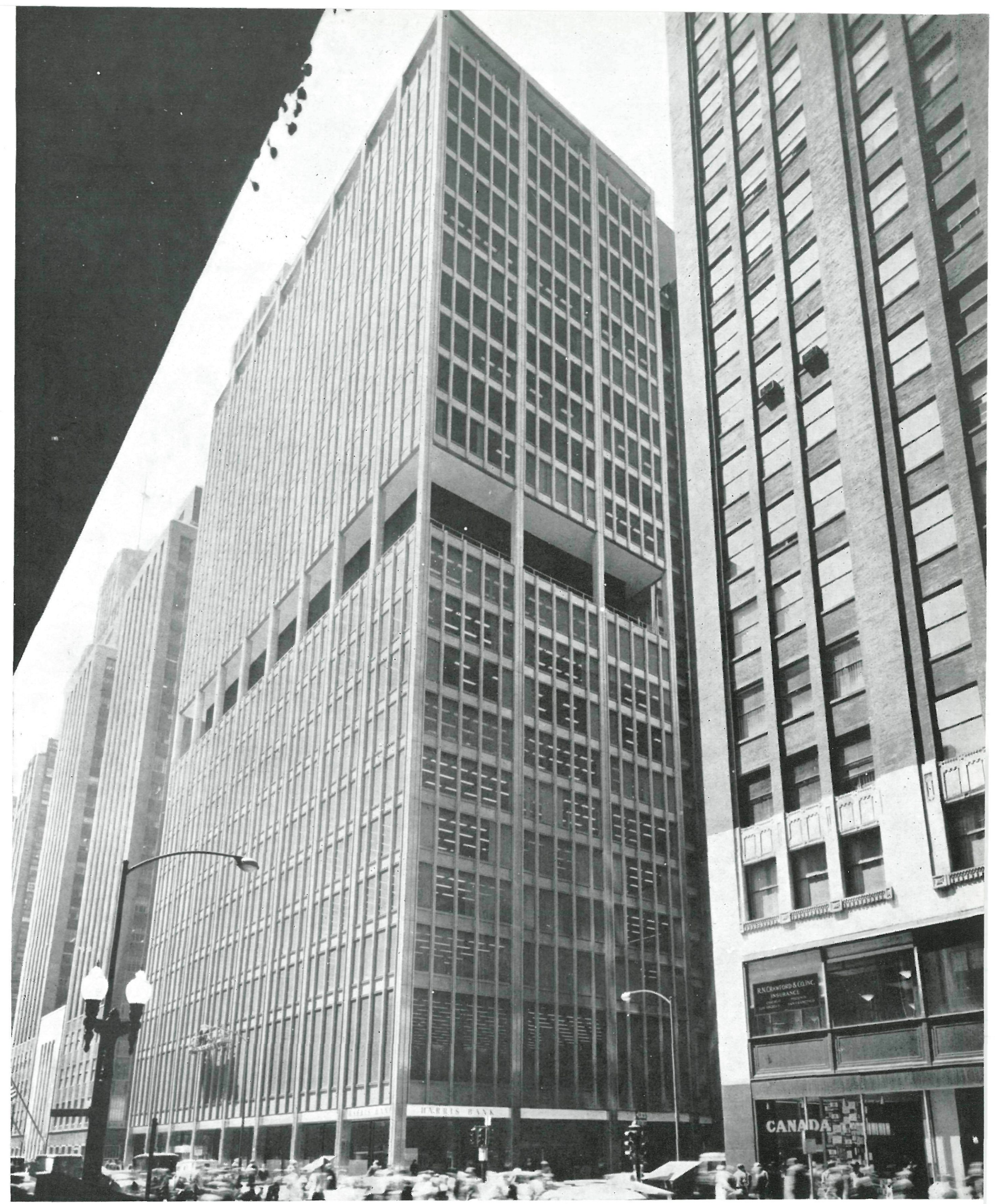

\title{
Edificio del trust Harris y banco Savings
}

SKIDMORE, OWINGS Y MERRILL, arquitectos ingenieros

$145 \cdot 55$ 

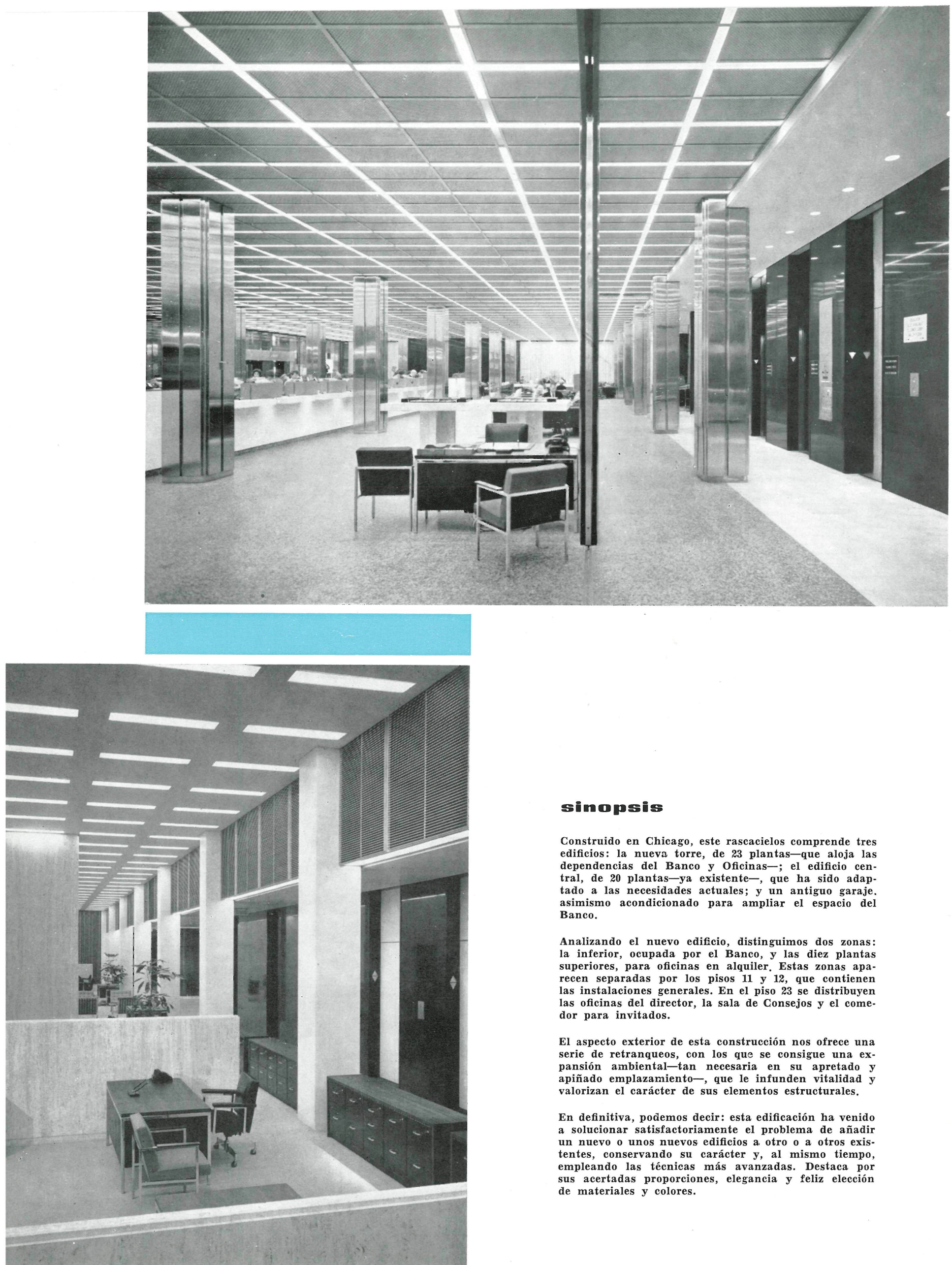

\section{simopsis}

Construido en Chicago, este rascacielos comprende tres edificios: la nueva torre, de 23 plantas-que aloja las dependencias del Banco y Oficinas-; el edificio central, de 20 plantas-ya existente-, que ha sido adaptado a las necesidades actuales; y un antiguo garaje. asimismo acondicionado para ampliar el espacio del Banco.

Analizando el nuevo edificio, distinguimos dos zonas: la inferior, ocupada por el Banco, y las diez plantas superiores, para oficinas en alquiler. Estas zonas aparecen separadas por los pisos 11 y 12 que contienen las instalaciones generales. En el piso 23 se distribuyen las oficinas del director, la sala de Consejos y el comedor para invitados.

El aspecto exterior de esta construcción nos ofrece una serie de retranqueos, con los que se consigue una expansión ambiental-tan necesaria en su apretado y piñado emplazamiento- que le infunden vitalidad valorizan el carácter de sus eler

En definitiva, pođemos decir: esta edificación ha venido a solucionar satisfactoriamente el problema de añadir un nuevo o unos nuevos edificios a otro o a otros existentes, conservando su carácter $y$, al mismo tiempo, empleando las técnicas más avanzadas. Destaca por sus acertadas proporciones, elegancia y feliz elección de materiales y colores. 
Este rascacielos, uno de los más modernos de Chicago, comprende tres edificios: la nueva torre, de 23 plantas, dedicada a dependencias del Banco y oficinas; el edificio central, de 20 plantas, ya existente, ocupado desde hace cincuenta años por el Banco, que ha sido reconstruido y adaptado a las funciones actuales; y un antiguo garaje-parking, asimismo acondicionado para ampliar el espacio del Banco.

El emplazamiento del nuevo edificio vino impuesto por las exigencias del extenso programa de necesidades y después de estudiar varias soluciones.

De todas ellas se eligió una que presenta las siguientes ventajas: poder colocar el nuevo edificio-torre en una esquina privilegiada; lograr un máximo aprovechamiento de la superficie construida; minimizar el espacio de pasillos, y obtener una óptima área perimetral para oficinas.

Analizando la nueva edificación, se distinguen dos zonas bien diferenciadas: la inferior, ocupada por el Ban$\mathrm{co}$, y las diez plantas superiores, destinadas a oficinas en alquiler.
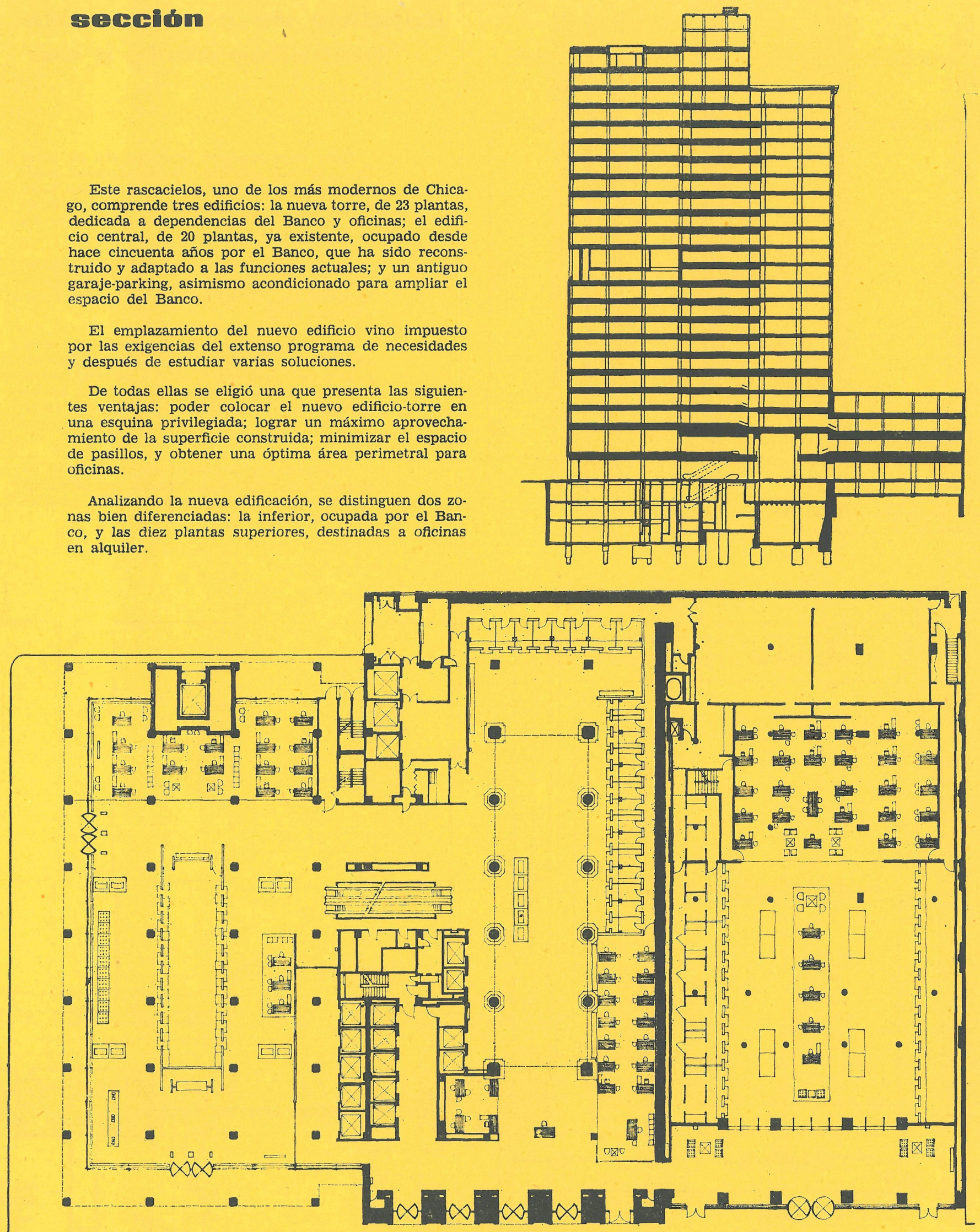

EDIFICIO NUEVO 


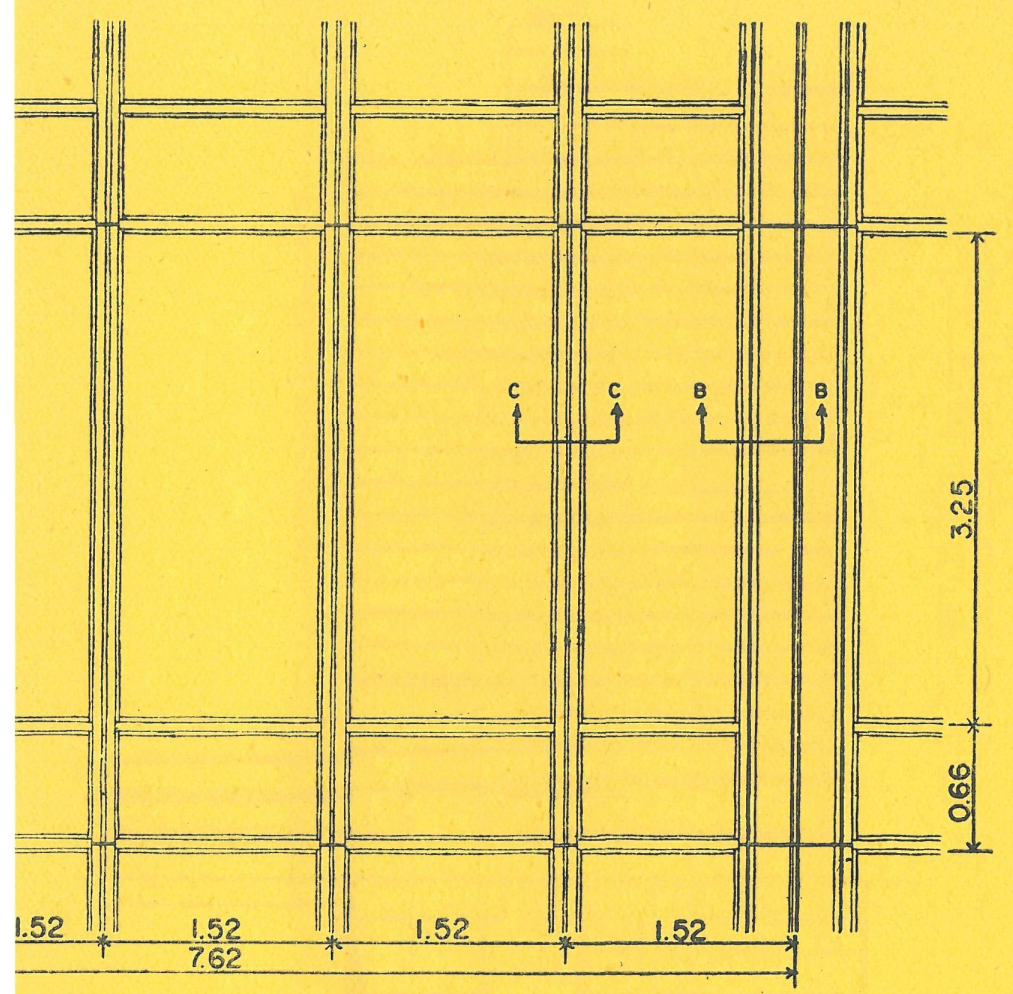

\section{detalles de cerramiento fachadas norte y sur}

Estas zonas aparecen separadas por los pisos $11 .^{\circ}$ y $12 .^{\circ}$ que contienen las instalaciones generales (aire acondicionado, etc.), las cuales constituyen una de las características constructivas de este edificio, ya que normalmente se disponen en la última planta.

El piso 23..$^{\circ}$ contiene las oficinas del Director, sala de Consejos y comedor para directivos e invitados; este último en conexión con su atractivo patio-jardín.

Las plantas han sido resueltas partiendo siem pre de la idea de unir en cada nivel los pisos del viejo y nuevo edificio, y crear un núcleo central - para economizar espacio-que contenga los ascensores, aseos y escaleras. Estas últimas, en algunos casos, son combinación de rampas y escaleras.

El aspecto exterior nos ofrece una serie de retranqueos, con los que se consigue una expansión ambiental muy necesaria en su apretado y apiñado emplazamiento.

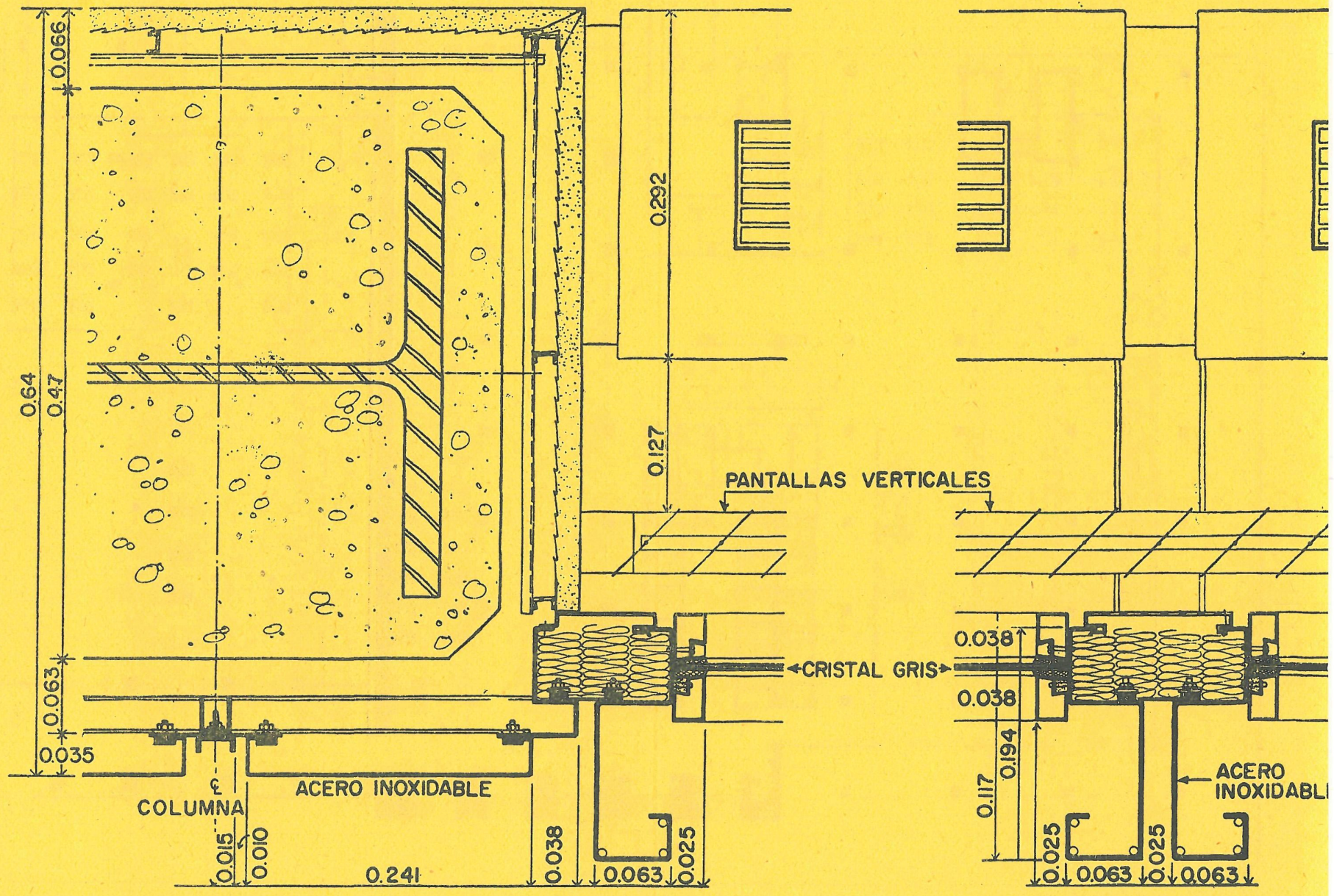




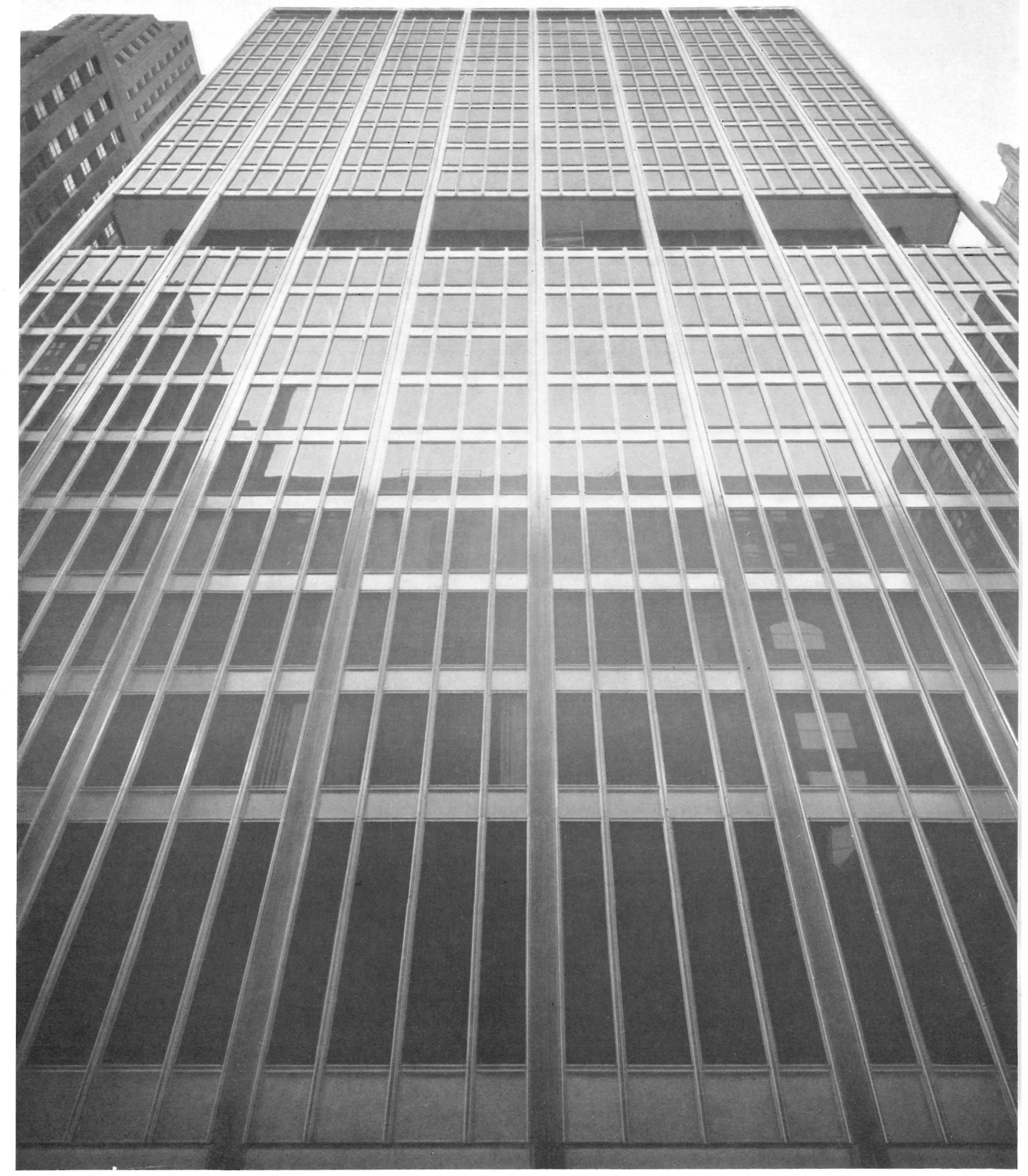

Estos retranqueos, que se presentan en planta baja, ático y pisos $11 .^{\circ}$ y $12 .^{\circ}$, rompen la monotonía del edificio, le infunden vitalidad y valorizan el carácter de los elementos estructurales.

En cuanto al sistema constructivo, se ha empleado el hormigón armado, tanto en muros como en pilares situados bajo la rasante del nivel de la calle; y estructura metálica en el resto del edificio, con los cerramientos a base de muros-cortina, en los que han sido utilizados acero inoxidable y cristal de color gris. 
En el interior los cielorrasos están cons tituidos por paneles de fibra de vidrio, ais lantes acústicos, sobre una retícula de aluminio, que presenta unos dispositivos que sirven, a la vez, para alojar los fluorescentes, y para inyectar o extraer el aire de las diversas dependencias.

En definitiva, la nueva torre del Trust Harris ha venido a solucionar uno de los problemas frecuentes en nuestro tiempo: añadir un nuevo o unos nuevos edificios, a otro o a otros existentes; reflejar sus necesidades; conservar su carácter y, al mismo tiempo, emplear las técnicas más modernas.

Podemos decir que destaca por su elegancia y acertadas proporciones, el cuidado con que han sido estudiados los detalles, y la feliz elección de materiales y colores.

Fotos: HEDRICH - BLESSING

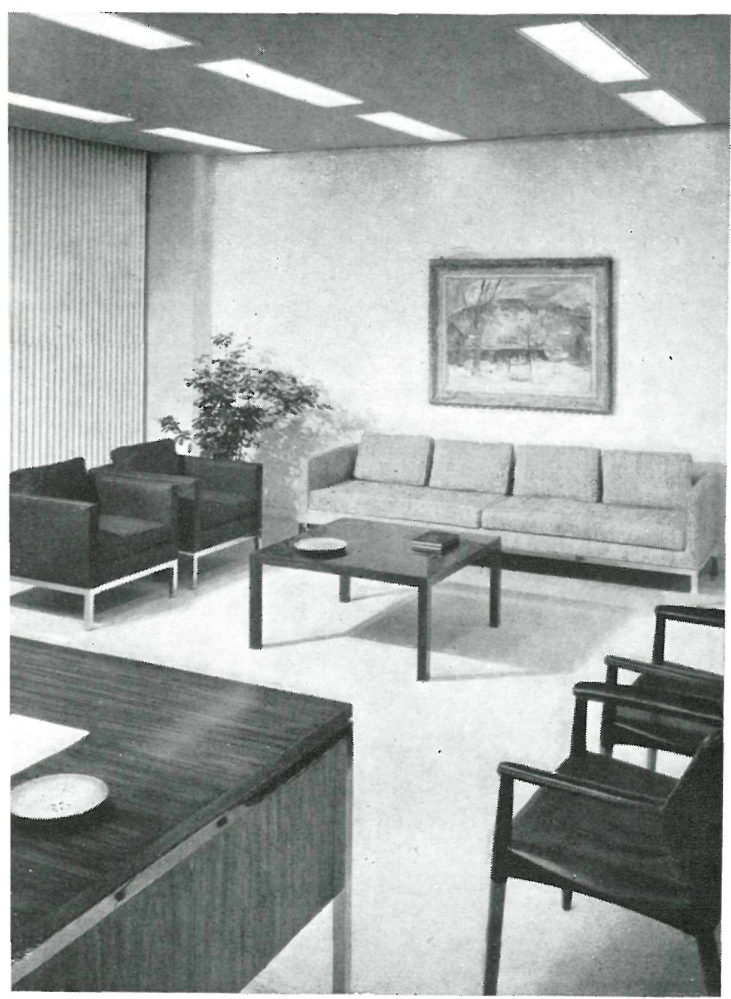

\section{interiores}
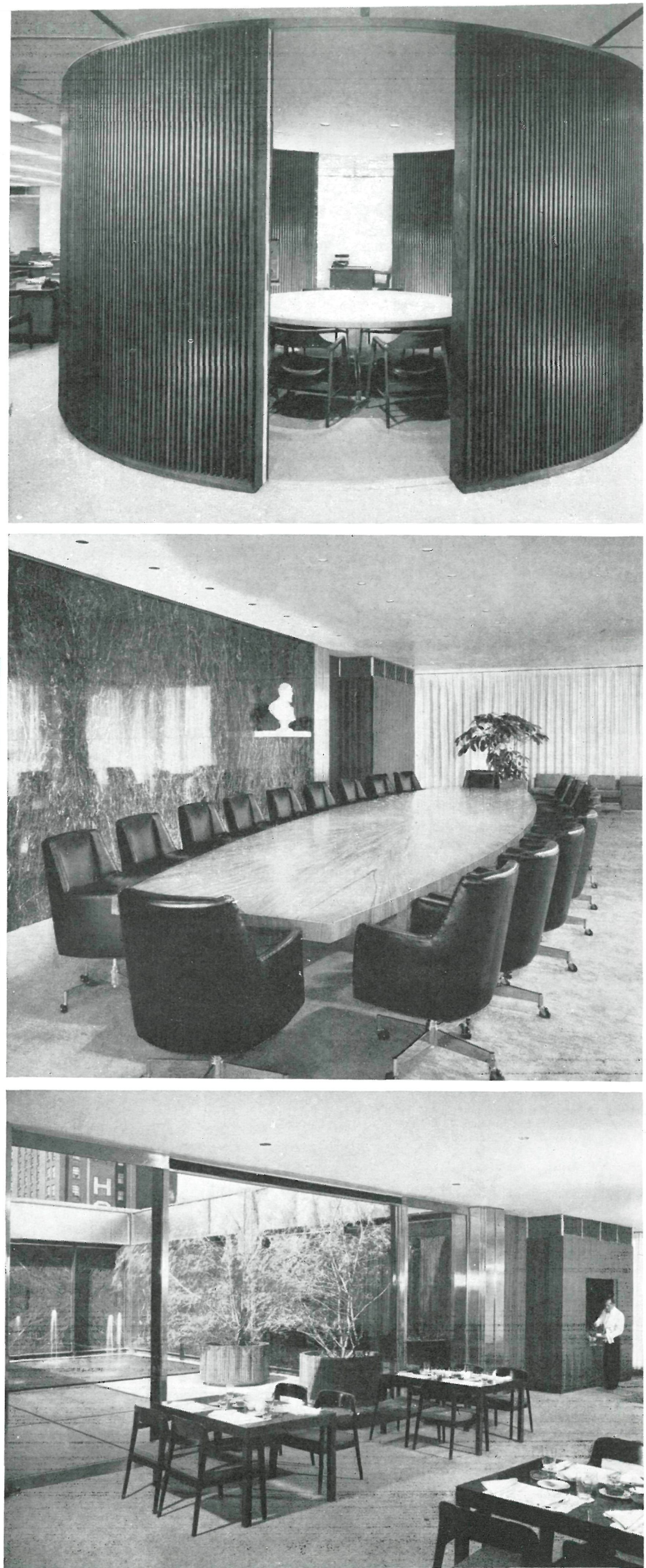


\section{Ediffice du Trust Haris et de Ir Bancume Savings}

Skidmore, Owings et Merrill, architectes-ingénieurs.

Construit à Chicago, ce gratte-ciel comprend trois édifices: la nouvelle tour, de 23 étages-qui abrite les dépenConstruit a Chicago, ce gratte-ciel complédifice central, de 20 étages-déjà existant-qui a été adapté aux nécesdances de la Banque et des bureaux-, et an etentent remanié pour l'agrandissement de la Banque.

L'analyse du nouvel édifice nous montre deux zones: l'inférieure, occupée par la Banque et les 10 étages supéL'analyse du nouvel édifice nous montre deux zones: l'inférieure, occupée par et le douzième étages qui abritent rieurs par des bureaux en location. seils et une salle à manger pour invités.

L'aspect extérieur de cette construction offre une série de retraits qui dégagent l'édifice, chose nécessaire étant donné sa situation dans une zone très resserrée et valorise le caractère de ses éléments structuraux.

En définitive on peut dire que cet édifice donne une solution satisfaisante au problème qui pose l'adjonction En définitive on peut dire que cet édifice donne une solution satisfaisante au problème qui poste leur caractère

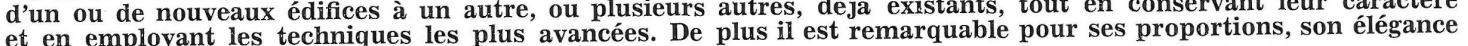
et le choix heureux de ses matériaux et couleurs.

\section{Hamis Trust and Savings Bamk Building}

Skidmore, Owings \& Merrill, architect engineers.

This large Chicago project consists of three structures: a new 23 storey block containing the bank and other offices; the central building, with 20 floor levels, recently adapted to present day requirements, and an old garage, also reconstructed and utilised by the bank.

The new building has two distinct functional zones: the lower part, occupied by the bank; and the top 10 storeys, which are let as office space. Between the two zones, floor levels 11 and 12 are devoted to general services and installations. On floor 23 the director has his office, and there is also space for a council room and a dining hall for guests.

Externally, the building is in the form of interesting and contrasting planes and volumes. This gives it a more expansive aspect, which is most necessary owing to the closely built area where it is situated, and enhaces an impression of vitality and positive interection between its structural elements.

As a whole, this building constitutes a satisfactory solution of the problem of adding a new building to two other already existant ones, so that the character of the latter is maintained, although new techniques are adopted. This project is also noteworthy because of its proportions, elegance and right choice of materials and colour.

\section{Geboiude der Trust Haris und Savings - Bank}

Skidmore, Owings und Merrill, Architekten-Ingenieure.

Dieser in Chicago erbaute Wolkenkratzer umfasst drei Gebäude: den neuen Turm mit 23 Stockwerken, in dem die Abteilungen der Bank und Bureauräume untergebracht sind; das mittlere Gebäude mit 20 bereits bestedie Abteilungen der Bank und Begenwärtigen Bedürfnissen angepasst worden ist; der alten Garage, ebenfalls für die Erweiterung der Bankräumlichkeiten hergerichtet.

Beim Aufgliedern des neuen Gebäudes unterscheiden wir zwei Zonen: die untere von der Bank belegte und die zehn oberen Geschosse zum Vermieten von Bureauräumen. Diese Zonen werden durch den 11. und 12. Stock getrennt, die die allgemeinen Installationen enthalten. Im 23. Geschoss befinden sich die Bureauräume des Direktors, der Ratsaal und das Speisezimmer für Gäste.

Der äussere Anblick dieses Baues bietet uns eine Reihe von Abrückungen, mit denen man eine Ausdehnung

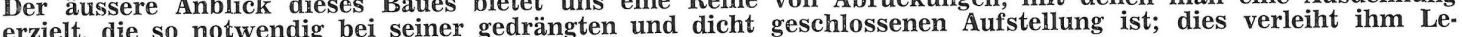
erzielt, die so notwenden Charakter seiner Strukturteile aus. Letzten Endes können wir sagen: dieses Gebäude hat zuffiedenstellend das Problem gelöst, ein neues oder einige neue Bauten an verhältnisse, Vornehmheit und glïckliche Auswahl der Materialien und Farben hervor. 